This item was submitted to Loughborough's Research Repository by the author.

Items in Figshare are protected by copyright, with all rights reserved, unless otherwise indicated.

\title{
Sharp bursts of high-flux reactive species in submicrosecond atmospheric pressure glow discharges
}

PLEASE CITE THE PUBLISHED VERSION

PUBLISHER

(C) American Institute of Physics

\section{VERSION}

VoR (Version of Record)

\section{LICENCE}

CC BY-NC-ND 4.0

\section{REPOSITORY RECORD}

Walsh, James L., and Michael G. Kong. 2019. "Sharp Bursts of High-flux Reactive Species in Submicrosecond Atmospheric Pressure Glow Discharges”. figshare. https://hdl.handle.net/2134/5190. 
This item was submitted to Loughborough's Institutional Repository (https://dspace.lboro.ac.uk/) by the author and is made available under the following Creative Commons Licence conditions.

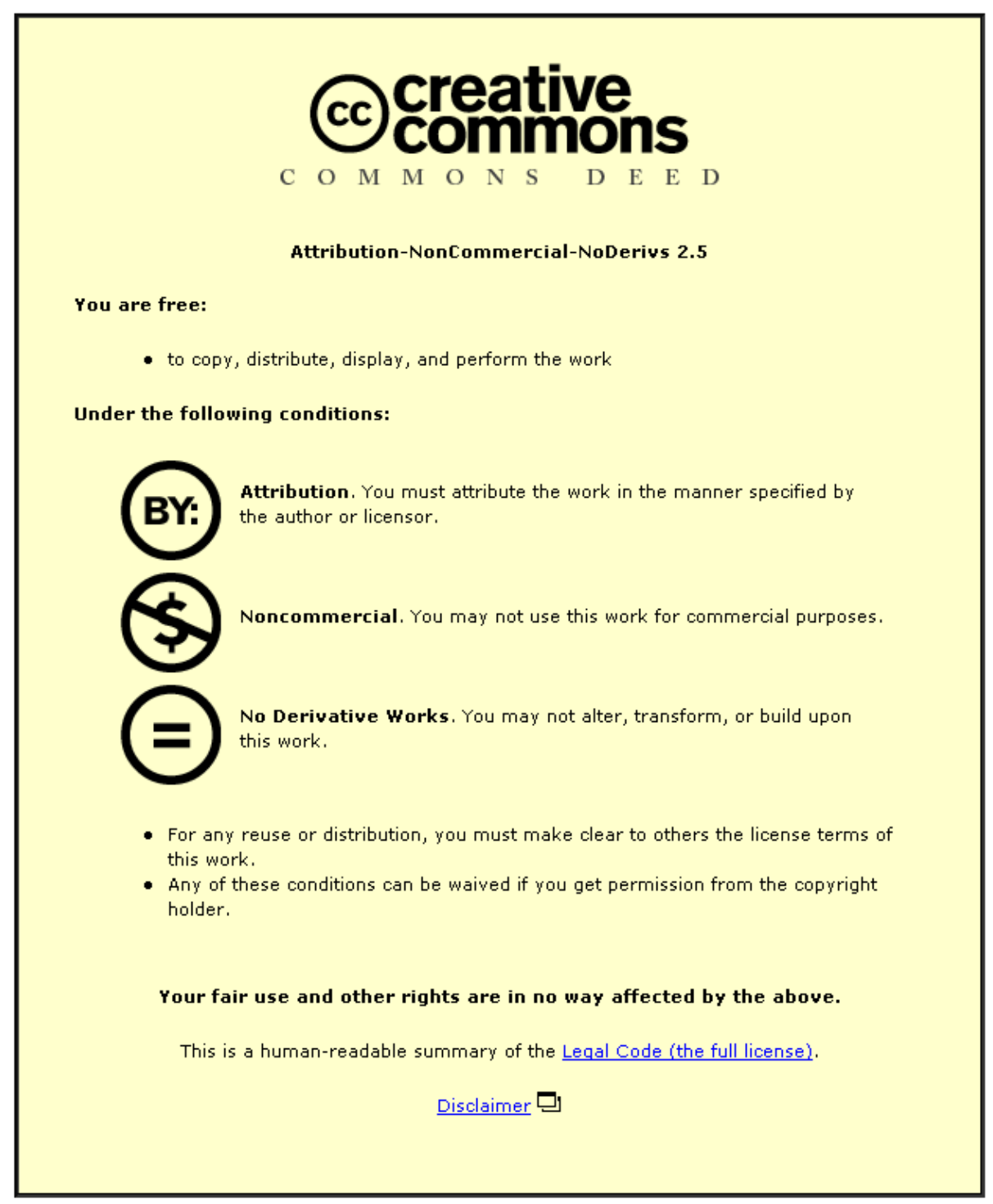

For the full text of this licence, please go to: http://creativecommons.org/licenses/by-nc-nd/2.5/ 


\title{
Sharp bursts of high-flux reactive species in submicrosecond atmospheric pressure glow discharges
}

\author{
J. L. Walsh and M. G. Kong ${ }^{\text {a) }}$ \\ Department of Electronic and Electrical Engineering, Loughborough University, Loughborough, \\ Leicestershire LE11 3TU, United Kingdom
}

(Received 31 August 2006; accepted 14 October 2006; published online 8 December 2006)

\begin{abstract}
In this letter, the authors present an experimental study of the temporal characteristics of submicrosecond pulsed atmospheric glow discharges. Using electrical measurements and nanosecond-resolved optical emission spectroscopy, they show that a long initial period of each voltage pulse is spent building up space charges and is then followed by a large current pulse in the voltage-falling phase. Reactive plasma species such as oxygen atoms and $\mathrm{OH}$ radicals are produced in a train of sharp and independent pulses of 50-100 ns wide. Finally, their production is shown to increase significantly as the voltage pulse width reduces or the repetition frequency increases. (C) 2006 American Institute of Physics. [DOI: 10.1063/1.2397570]
\end{abstract}

With an immense application potential, atmospheric pressure glow discharges (APGDs) have recently commanded much attention. ${ }^{1}$ A vast majority of APGD are generated with sinusoidal excitation at frequencies of typically $1-100 \mathrm{kHz}$ (Refs. 2-5) and 1-60 MHz. ${ }^{6-10}$ Much less studied are APGD generated by high-voltage pulses with pulse width of $0.5-100 \mu \mathrm{s}$ and at a repetition frequency of a few kilohertz. ${ }^{11-14}$ Compared to sinusoidal APGD, pulsed APGD tend to have low gas temperature, ${ }^{11,14}$ high discharge current, ${ }^{12-14}$ and high electrical efficiency. ${ }^{11,14}$ They are particularly desirable for applications where wall-plug efficiency or/and room-temperature processing are critical, for example industrial-scale surface treatment ${ }^{15}$ and food decontamination. ${ }^{16}$ In a recent study, we reported a $1 \mathrm{kHz}$ APGD generated with $360 \mathrm{~ns}$ high-voltage pulses between two naked electrodes. ${ }^{17}$ With a large current density of $0.8 \mathrm{~A} / \mathrm{cm}^{2}$, the pulsed APGD was shown to produce far more intense atomic oxygen emission line at $777 \mathrm{~nm}$ than a comparable atmospheric dielectric-barrier discharge (DBD). ${ }^{17}$ This is significant, given that reactive species such as atomic oxygen and $\mathrm{OH}$ radicals are key application enablers. ${ }^{14-16,18}$ Therefore, it is of considerable interest to better understand and further develop pulsed APGD. In this letter, we present an experimental study of temporal characteristics of submicrosecond pulsed APGD and their generation of reactive plasma species under different pulsing conditions.

The submicrosecond pulsed APGD used for this investigation employed two parallel stainless-steel electrodes with a surface area of $6 \mathrm{~cm}^{2}$ and a separation distance of $0.5 \mathrm{~cm}$ nominal. The two electrodes were naked without any dielectric barriers, and the electrode unit was housed within a Perspex box fed with a through flow of helium-oxygen mixture. Helium flow rate was fixed at 7 SLM (standard liters per minute) and oxygen flow rate at 10 SCCM (SCCM denotes cubic centimeter per minute at STP). The Perspex box was not air tight, and so ambient oxygen and nitrogen were present. A homemade power source provided a train of unipolar voltage pulses of up to $15 \mathrm{kV}$ at $0.01-20 \mathrm{kHz}$. The

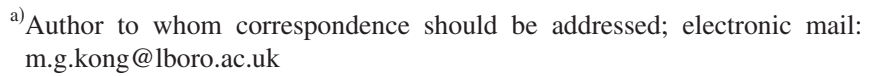

peak value of the voltage pulses was an adjustable input parameter, and the pulse duration was determined largely by the interaction of the power supply with the plasma. When the plasma current became sufficiently large, it increased the electrical conductivity of the gas gap critically to cause a sharp fall of the output voltage. Current and voltage were measured with a wideband current probe (Tektronix P6021), a wideband voltage probe (Tektronix P6015A), and a highspeed oscilloscope (Tektronix TDS 3000B). To allow for nanosecond-resolved spectral measurement, a pulse generator was used for triggering. Optical emission spectra were measured by an Andor Shamrock spectrometer with a focal length of $0.3 \mathrm{~m}$ and a grating of 600 grooves $/ \mathrm{mm}$.

Figure 1(a) shows electrical measurements of a $4 \mathrm{kHz}$ submicrosecond pulsed APGD. The mean peak voltage is $V_{p}=2.4 \mathrm{kV}$, the peak discharge current is $I_{p}=8.4 \mathrm{~A}$, and the peak dissipated power is $13.7 \mathrm{~kW}$. Similar to those reported previously, ${ }^{17}$ nanosecond plasma images suggest that the pulsed atmospheric plasma was spatially uniform without unstable streamers and its spatial structure had a clear sheath region (image not shown). Electrode temperature measured with a thermocouple was found to increase about $2{ }^{\circ} \mathrm{C}$ above room temperature. These observations confirm that it was an atmospheric pressure glow discharge. Compared to sinusoidal atmospheric DBD and indeed radio-frequency APGD, the pulsed APGD can be operated at very high peak power

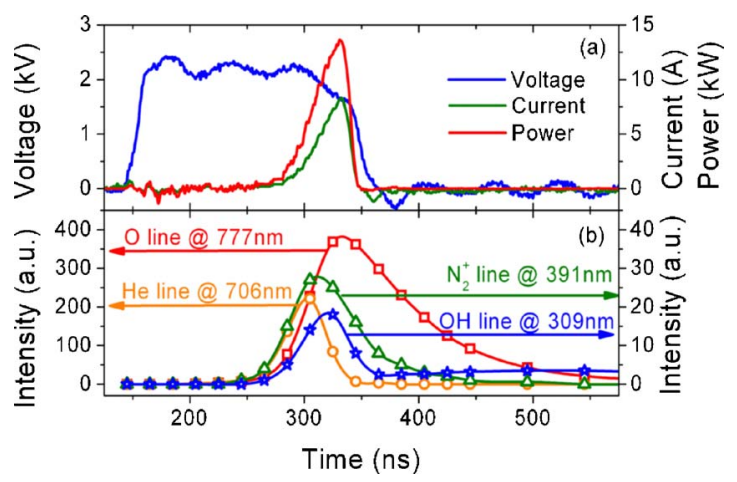

FIG. 1. (Color online) Characteristics of a submicrosecond APGD with (a) electrical measurement and (b) nanosecond-resolved optical emission of key plasma species. 


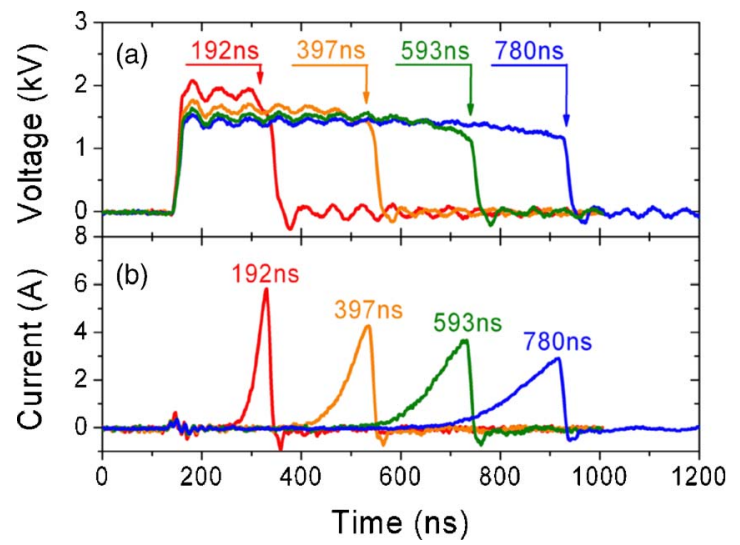

FIG. 2. (Color online) (a) Applied voltage and (b) discharge current of a submicrosecond APGD with four different voltage pulse widths.

density of $1-5 \mathrm{~kW} / \mathrm{cm}^{3}$ without inducing any significant temperature rise. Figure 1(a) shows that its voltage pulse has a rise time of $18 \mathrm{~ns}$ and a fall time of $55 \mathrm{~ns}$, both measured between $10 \%$ and $90 \%$ of the peak voltage, and a pulse width of $\Delta_{v}=190 \mathrm{~ns}$ measured as the full width at half maximum. The current pulse has a rise time of $43 \mathrm{~ns}$, a fall time of $10 \mathrm{~ns}$, and a pulse width of $\Delta_{c}=30 \mathrm{~ns}$. With a voltage risetime of $18 \mathrm{~ns}$ and a rig capacitance of $1.1 \mathrm{pF}$, the peak displacement current is $0.15 \mathrm{~A}$ and much less than the peak current of $8.4 \mathrm{~A}$. Therefore, the discharge current shown in Fig. 1(a) is likely to be the conduction current.

The discharge current pulse occurs in the voltage-falling phase, most probably because the electric field setup during the voltage-rising phase is insufficient to trigger gas ionization. ${ }^{17}$ For helium APGD, it has been reported that the electric field must exceed a threshold of about $5.9 \mathrm{kV} / \mathrm{cm}$ in order to compensate electrons lost with electrons created. ${ }^{19}$ During the voltage-rising phase, the peak electric field is unlikely to be much above $2.4 \mathrm{kV} / 0.5 \mathrm{~cm}=4.8 \mathrm{kV} / \mathrm{cm}$. Markedly below the $5.9 \mathrm{kV} / \mathrm{cm}$ threshold, this is insufficient to trigger electron avalanche and so explains why no current peak is observed in the voltage-rising phase. It is worth mentioning that the short voltage pulse width prevents the generated electrons from reaching the anode. Assuming a peak electric field of $4.4 \mathrm{kV} / \mathrm{cm}$ and an electron mobility of $1132 \mathrm{~cm}^{2} \mathrm{~V}^{-1} \mathrm{~s}^{-1}$ for the helium-oxygen mixture, ${ }^{17}$ the time for an electron to cross the $0.5 \mathrm{~cm}$ electrode gap is found to be $100 \mathrm{~ns}$ - about $53 \%$ of the voltage pulse width. During the initial period of $100 \mathrm{~ns}$, the generated electrons are trapped in the electrode gap and contribute to the buildup of space charges. This charge-accumulating period of $100 \mathrm{~ns}$ is similar to the time difference of $\Delta t_{\text {onset }}=127 \mathrm{~ns}$ in the onset instant between the voltage and current pulses. In other words, a large portion of the voltage pulse is spent building up space charges so that the maximum electric field is raised above the breakdown electric field and triggers an electron avalanche.

The above discussion is supported by results of optical emission spectroscopy. It is known that the $391 \mathrm{~nm}$ line contains the optical signature of helium metastables and molecular helium ions. ${ }^{20}$ It is also known that the $706 \mathrm{~nm}$ line is linked to either energetic electrons or $\mathrm{He}_{2}^{+}$and low energy electrons. ${ }^{20}$ As the most numerous ions in helium APGD are molecular helium ions, ${ }^{21}$ the temporal profiles of the 391 and $706 \mathrm{~nm}$ lines follow the profile of space charges. As shown in Fig. 1(b), emission line intensities at 391 and $706 \mathrm{~nm}$ are Downloaded 21 Aug 2009 to 158.125 .80 .71 . Redistribution subject

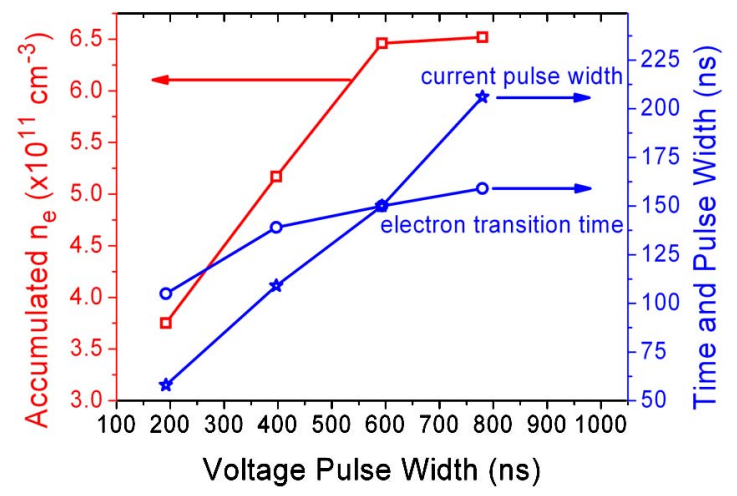

FIG. 3. (Color online) Pulse width dependence of the time-accumulated electron density over $1 \mathrm{~V}$ pulse, the electron transition time across the electrode gap, and the current pulse width measured between two $0.1 I_{p}$ points.

negligible in the first $122 \mathrm{~ns}$ of the voltage pulse and so initially there are very few space charges in the gas gap to sustain a large current. The negligible emission period of $122 \mathrm{~ns}$ agrees very well with $\Delta t_{\text {onset }}=127 \mathrm{~ns}$.

Figure 1(b) also shows a pulse of the $\mathrm{OH}$ line at $309 \mathrm{~nm}$ and a pulse of the oxygen atom line at $777 \mathrm{~nm}$, with their pulse widths being 60.0 and $106.3 \mathrm{~ns}$, respectively. When compared to an atmospheric DBD using the same electrode unit, the same background gas and a very similar peak voltage, the intensities of the 307 and $777 \mathrm{~nm}$ emission lines in the pulsed APGD are up to ten times greater (data not shown). Therefore, a distinct character of submicrosecond pulsed APGD is their ability to produce high fluxes of reactive plasma species such as oxygen atoms and $\mathrm{OH}$ radicals. At a repetition frequency of $4 \mathrm{kHz}$, the half period is $125 \mu \mathrm{s}$ and much longer than the electron transition time of $\tau_{e}$ $=100 \mathrm{~ns}$ across the electrode gap. Therefore, electrons generated in one voltage pulse are most certainly lost to the electrodes and through ionic recombination well before the next voltage pulse. This suggests that reactive plasma species such as oxygen atoms and $\mathrm{OH}$ radicals are generated as a train of sharp and independent bursts. It is conceivable that such submicrosecond windows of high-flux reactive species could facilitate surface modification with spatial or/and temporal selectivity. For example, a surface pattern with sharp boundaries could be realized by moving a pulsed APGD jet over a surface at a speed that translates the time scale of the windows to spatial dimensions of the surface pattern.

To enhance the production of reactive plasma species, we altered the peak applied voltage so as to alter the discharge current. As the peak applied voltage increases, Fig. 2 shows that the peak discharge current increases but the voltage pulse width decreases. The peak current $I_{p}$ is very high, ranging from 3 to $6 \mathrm{~A}$, and corresponds to a current density of $0.5-1.0 \mathrm{~A} / \mathrm{cm}^{2}$. It is probable that such a high discharge current could substantially increase electrical conductivity of the gas to short-circuit the gas gap, leading to a premature shortening of the voltage pulse. To study this further, we integrated the current pulses in Fig. 2(b) to obtain the total accumulated electron density generated during each voltage pulse. This is shown in Fig. 3 together with the electron transition time across the electrode gap $\tau_{e}$ and the current pulse width $\Delta_{c}$. the latter now being measured between the two $0.1 I_{p}$ points to be comparable to the accumulated electron density that is obtained by integrating over the entire current pulse. While the voltage pulse width varies by a facto AIP license or copyright; see http://apl.aip.org/apl/copyright.jsp 


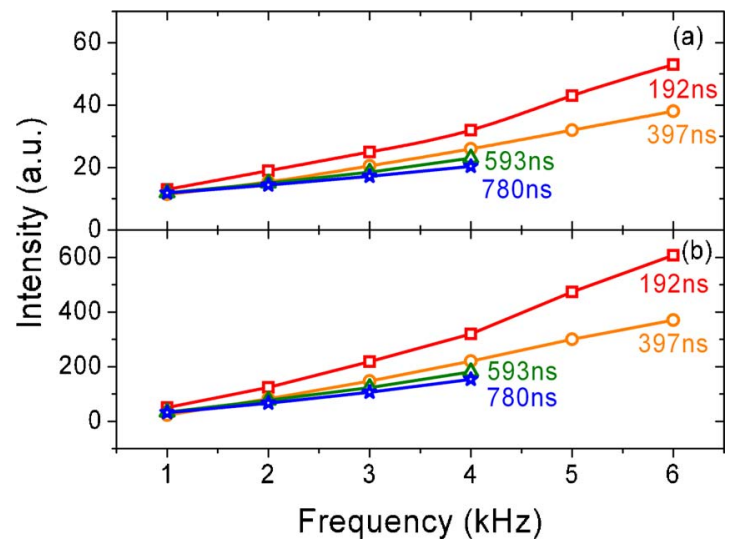

FIG. 4. (Color online) Frequency dependence of emission intensity of (a) the $\mathrm{OH}$ line at $309 \mathrm{~nm}$ and (b) the atomic oxygen line at $777 \mathrm{~nm}$.

tor of more than 4 , the total accumulated electron density varies only by a factor of 1.7 suggesting similar gas conductivity among all four cases. At $\Delta_{v}=192 \mathrm{~ns}, \tau_{e}$ is $106 \mathrm{~ns}$ and $\Delta_{c}$ is $58 \mathrm{~ns}$. Therefore during the current pulse, most generated electrons are unable to reach the anode and so are trapped in the gas gap. In this case, the accumulated electron density is likely to be close to the peak electron density of $3.6 \times 10^{11} \mathrm{~cm}^{-3}$ in the gas gap. At $\Delta_{v}=397 \mathrm{~ns}, \tau_{e}=139 \mathrm{~ns}$ and $\Delta_{c}=109$ ns. As $\tau_{e}<\Delta_{c}$ and they are now comparable, many generated electrons are trapped in the gas gap but the trapped electrons are proportionally fewer than those in the $\Delta_{v}=192 \mathrm{~ns}$ case. As a result, the peak electron density achieved during the current pulse is smaller than the accumulated electron density of $5.1 \times 10^{11} \mathrm{~cm}^{-3}$ in Fig. 3. As $\Delta_{v}$ increases to $593 \mathrm{~ns}$, both $\tau_{e}$ and $\Delta_{c}$ become $150 \mathrm{~ns}$. This suggests that a significant proportion of generated electrons are now lost to the electrodes and the peak electron density reached during the current pulse is likely to be markedly smaller than the accumulated electron density of 6.5 $\times 10^{11} \mathrm{~cm}^{-3}$. This difference is greater in the case of $\Delta_{v}$ $=780 \mathrm{~ns}$, for which $\tau_{e}=159 \mathrm{~ns}$ is now less than $\Delta_{c}=206 \mathrm{~ns}$. Therefore as $\Delta_{v}$ increases, the peak electron density achieved during a current pulse becomes progressively less than its corresponding accumulated electron density in Fig. 3. This suggests that the peak electron density and hence the maximum electrical conductivity of the gas gap change little during the current pulse for all four cases of Fig. 3. It is therefore highly likely that narrow voltage pulse width at large voltage is caused by plasma-induced short circuiting of the gas gap.

Figure 4 shows that the optical emission intensity of $\mathrm{OH}$ line at $309 \mathrm{~nm}$ and atomic oxygen line at $777 \mathrm{~nm}$ for the four different voltage pulse widths. At a fixed frequency, stronger emission intensity is achieved with shorter pulse width that is linked to a large peak current (see Fig. 2). At a given $\Delta_{v}$, the emission intensity increases with increasing frequency. At $\Delta_{v}=192 \mathrm{~ns}$, the $777 \mathrm{~nm}$ line intensity increases by a factor of 12 as the frequency increases from 1 to $6 \mathrm{kHz}$. A high frequency leads to more frequent production of sharp bursts of reactive plasma species and so to their greater accumulation over a given time period. Also at long voltage pulsewidth of $\Delta_{v}=593$ and $780 \mathrm{~ns}$, stable APGDs are achieved only at frequencies up to $4 \mathrm{kHz}$. If $\Delta_{v}$ is reduced to below $400 \mathrm{ns,}$

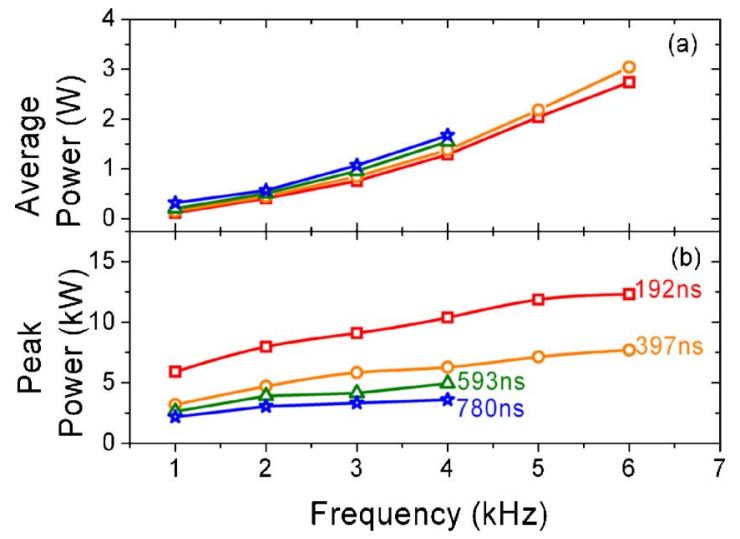

FIG. 5. (Color online) Frequency dependence of (a) the average dissipated power and (b) the peak electrical power.

stable APGD can be extended to above $6 \mathrm{kHz}$.

To place the above comparison in the context of energy budget, the average dissipated power is shown in Fig. 5 to increase with the frequency but change little with voltage pulse width. Combining this result with those in Fig. 4, it is clear that production of oxygen atoms and $\mathrm{OH}$ radicals can be increased substantially without consuming more electrical power. The peak power is very large, particularly with short voltage pulse width. This is useful to sustain a large current and a large flux of reactive plasma species.

This work was funded in part by the Engineering and Physical Sciences Research Council, UK.

${ }^{1}$ J. R. Roth, Industrial Plasma Engineering (Institute of Physics, Philadelphia, 1995), Vol. 1, pp. 453-460.

${ }^{2}$ S. Kanazawa, M. Kogoma, T. Moriwaki, and S. Okazaki, J. Phys. D 21, 838 (1988).

${ }^{3}$ F. Massines and G. Gouda, J. Phys. D 31, 3411 (1998).

${ }^{4}$ I. Radu, R. Bartnikas, and M. R. Wertheimer, IEEE Trans. Plasma Sci. 31, 411 (2003).

${ }^{5}$ X. T. Deng and M. G. Kong, IEEE Trans. Plasma Sci. 32, 1709 (2004).

${ }^{6}$ S. E. Babayan, J. Y. Jeong, V. J. Tu, J. Park, G. S. Selwyn, and R. F. Hicks, Plasma Sources Sci. Technol. 7, 286 (1998).

${ }^{7}$ J. J. Shi, X. T. Deng, R. Hall, J. D. Punnet, and M. G. Kong, J. Appl. Phys. 94, 6303 (2003).

${ }^{8}$ L. Baars-Hibbe, C. Schrader, P. Sichler, T. Cordes, K. H. Gericke, S. Buttgenbach, and S. Draeger, Vacuum 73, 327 (2004).

${ }^{9}$ Z. Yu, K. Hoshimiya, J. D. Williams, S. F. Polvinen, and G. J. Collins, Appl. Phys. Lett. 83, 854 (2003).

${ }^{10}$ J. J. Shi and M. G. Kong, Phys. Rev. Lett. 96, 105009 (2006).

${ }^{11}$ M. G. Kong and X. T. Deng, IEEE Trans. Plasma Sci. 31, 7 (2003).

${ }^{12}$ M. Laroussi, X. Liu, V. Kolobov, and R. Arslanbekov, J. Appl. Phys. 96, 3028 (2004).

${ }^{13}$ M. Laroussi, X. Liu, V. Kolobov, and R. Arslanbekov, J. Appl. Phys. 96, 3028 (2004).

${ }^{14}$ J. L. Walsh, J. J. Shi, and M. G. Kong, Appl. Phys. Lett. 88, 171501 (2006).

${ }^{15}$ J. R. Roth, Phys. Plasmas 12, 057103 (2005).

${ }^{16}$ M. Vleugels, G. Shama, X. Deng, E. Greenacre, T. Brocklehurst, and M. G. Kong, IEEE Trans. Plasma Sci. 33, 824 (2005).

${ }^{17}$ J. L. Walsh, J. J. Shi, and M. G. Kong, Appl. Phys. Lett. 89, 161505 (2006).

${ }^{18}$ M. Moravej, X. Yang, R. F. Hicks, J. Penelon, and S. E. Babayan, J. Appl. Phys. 99, 093395 (2006).

${ }^{19}$ J. Shi and M. G. Kong, Appl. Phys. Lett. 86, 091502 (2005).

${ }^{20}$ G. Nersisyan and W. G. Graham, Plasma Sources Sci. Technol. 13, 582 (2004).

${ }^{21}$ J. J. Shi and M. G. Kong, J. Appl. Phys. 97, 023306 (2005). 